\title{
Ein Zwischenfall, dem Heinz Hopf 1939 in Karlsruhe ausgesetzt war
}

\section{Journal Article}

Author(s):

Stammbach, Urs

Publication date:

2009

Permanent link:

https://doi.org/10.3929/ethz-b-000422880

Rights / license:

In Copyright - Non-Commercial Use Permitted

Originally published in:

Mathematische Semesterberichte 56(2), https://doi.org/10.1007/s00591-009-0058-6 


\section{Ein Zwischenfall, dem Heinz Hopf 1939 in Karlsruhe ausgesetzt war}

\section{Urs Stammbach}

Eingegangen: 20. April 2009 / Angenommen: 9. Mai 2009 / Online: 3. Juni 2009

(C) Springer-Verlag 2009

Zusammenfassung Am 9. Januar 1939 wurde Heinz Hopf, damals ordentlicher Professor für Mathematik an der ETH in Zürich, auf der Rückreise aus Berlin in Karlsruhe von der Gestapo verhaftet und in Untersuchungshaft genommen. Die dramatischen Umstände dieser gefährlichen Verwicklung lassen sich dank der heute im Archiv der Bibliothek der ETH vorhandenen Unterlagen in großen Zügen rekonstruieren. Darunter befinden sich auch Unterlagen, die dem Archiv erst vor kurzer Zeit von Dr. Klaus Völlm zur Verfügung gestellt worden sind. Es ergibt sich daraus ein beklemmendes Bild der Umstände, in denen Personen und Institutionen damals Entscheidungen haben fällen müssen.

\section{Heinz Hopfs Bemühen um die Einreise seines Vaters}

Heinz Hopf (siehe Abb. 1) war im Jahre 1931 als ordentlicher Professor für Mathematik von Berlin an die ETH Zürich berufen worden. Er vertrat die sich damals ganz neu entwickelnde algebraische Topologie. Als sehr erfolgreicher Forscher und Lehrer baute er an der ETH in den dreißiger Jahren rasch eine hervorragende Schule auf, aus der im Laufe der Jahre eine große Anzahl von weltweit führenden Topologen hervorgingen. ${ }^{1}$

Hopfs Vater, Wilhelm Hopf, war jüdischer Abstammung, seine Kinder Hedwig und Heinz waren somit im Nazi-Jargon „Halbjuden“; beide waren evangelisch getauft. ${ }^{2}$ Wilhelm Hopf lebte damals zusammen mit seiner Frau in Breslau. Es zeugt

U. Stammbach (『)

Mathematik, ETH-Zentrum, 8092 Zürich, Switzerland

E-Mail: stammb@math.ethz.ch

${ }^{1}$ Heinz Hopf war bis 1964 an der ETH tätig. Er starb 1971 in Zürich. (Siehe [3, 6].)

${ }^{2}$ Wilhelm Hopf wurde 1861 in Nürnberg als Sohn des Kaufmanns und Kommerzienrat Stephan Hopf geboren. Er war jüdischer Religon, trat dann aber 1895 zur evangelischen Kirche über. Von 1888 bis 


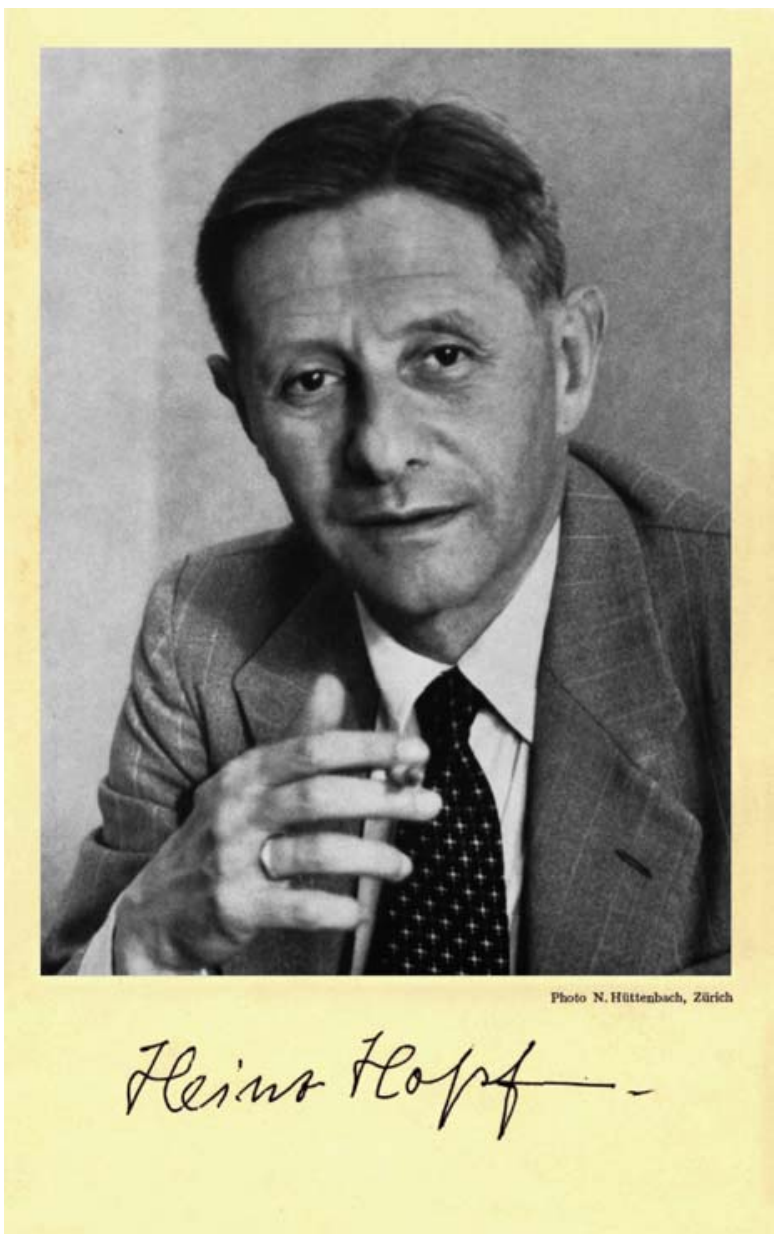

Abb. 1 Heinz Hopf (1884-1971)

für seine dortige gesellschaftliche Stellung, dass er in der Weimarer Republik das Ehrenamt eines Handelsgerichtsrates versah. Wegen seiner jüdischen Herkunft wurden seine Lebensumstände in der Zeit der Naziherrschaft immer schwieriger; trotz der Diskriminierungen und trotz des wiederholten Drängens seines Sohnes Heinz wollte er aber Deutschland vorerst nicht verlassen. Nachdem dann in der Reichspogromnacht vom 9. auf den 10. November 1938 in ganz Deutschland jüdische Geschäfte und Synagogen zerstört wurden und in den darauf folgenden Tagen zwischen 20.000 und 30.000 Juden verhaftet wurden, sah es sein Sohn in Zürich als seine Pflicht an, auch gegen den Willen seines Vaters aktiv zu werden und bei den Schweizer

1920 war er Brauereibesitzter in Breslau. Seit 1892 war er mit Elisabeth Kirchner, geboren 1872, der Tochter des Breslauer Brauereibesitzers Heinrich Kirchner verheiratet. Das Ehepaar hatte zwei Kinder, die Tochter Hedwig, 1893-1953 und den Sohn Wilhelm Heinrich, genannt Heinz, 1894-1971. 
Behörden eine Einreise- und Aufenthaltsbewilligung zu erwirken. ${ }^{3}$ Als erstes wandte sich Heinz Hopf am 11. November an den Schulratspräsidenten Arthur Rohn ${ }^{4}$ :

[...] seit den neuesten antisemitischen Exzessen in Deutschland halten meine Frau und ich es für unsere absolute Pflicht, alles, was in unseren Kräften steht, zu tun, um meine Eltern zur Annahme dieses Vorschlages [die Eltern bei sich aufzunehmen] zu bewegen und hauptsächlich: um die Ausführung des Vorschlages zu ermöglichen.

Er bitte daher um Hilfe, bei den Schweizerischen Behörden für seinen 77jährigen Vater und seine 66jährige Mutter eine Aufenthaltsbewilligung zu erwirken.

Bereits am 17. November konnte Schulratspräsident Rohn mitteilen, dass ihm Regierungsrat Briner ${ }^{5}$, Vorsteher der Polizei- und Militärdirektion des Kantons Zürich, zugesichert habe, dass dem Wunsch entsprochen werden könne. Er forderte Hopf auf, ein entsprechendes Gesuch unter Bezugnahme auf ihn direkt bei der kantonalen Fremdenpolizei einzureichen. Dieses Gesuch, seinen Eltern die Einreise in die Schweiz zu gestatten, trägt das Datum vom 20. November. Darin verpflichtet sich Hopf, die Eltern bei sich in Zollikon aufzunehmen und für ihren Unterhalt zu sorgen.

Das Gesuch wurde am 15. Dezember 1938 von der Eidgenössischen Fremdenpolizei bewilligt. Leider musste Heinz Hopf am 3. Februar 1939 mitteilen, dass sich die Reise der Eltern nicht habe durchführen lassen; er bitte um eine Verlängerung der Einreisebewilligung. Dies geschah mit Schreiben vom 16. Februar. Doch am 28. April musste Heinz Hopf die Fremdenpolizei informieren, dass sich die Auswanderung seiner Eltern wegen des Gesundheitszustandes des Vaters nun nicht mehr durchführen lasse, das Vorhaben müsse als ein zu großes Wagnis betrachtet werden. ${ }^{6}$

\section{Die Reise nach Breslau und Berlin und die Verhaftung in Karlsruhe}

Getrieben von Sorge um seine Eltern reiste Heinz Hopf zusammen mit seiner Frau ${ }^{7}$ über die Festtage 1938/39 zu seinen Eltern in Breslau. Die Rückreise Anfang Januar 1939 führte über Berlin, wo das Ehepaar Hopfs Schwester Hedwig und den Schwager Dr. Fritz Brinckmann ${ }^{8}$ besuchte. ${ }^{9}$ Heinz Hopf und seine Frau verbrachten die Tage ab

\footnotetext{
${ }^{3}$ Die Unterlagen zu diesem Abschnitt, aus denen hier zitiert wird, finden sich unter [7], Hs 622:30-42.

${ }^{4}$ Der Schweizerische Schulrat war zu jener Zeit das leitende Gremium der ETH in Zürich; er war direkt dem Bundesrat, dem höchsten Exekutivorgan der Schweiz unterstellt. Arthur Rohn (1878-1956) versah das Präsidentenamt von 1926 bis 1948.

${ }^{5}$ Dr. Robert Briner, 1885-1960, war von 1935 bis 1951 Mitglied des Regierungsrates des Kantons Zürich. Er hatte Rechtswissenschaft studiert und vertrat die Demokratische Partei.

${ }^{6}$ Wilhelm Hopf starb 1942 in Breslau. Seine Frau Elisabeth überlebte den Krieg. In einer offensichtlich abenteuerlichen Flucht, zu deren Einzelheiten sie selbst keine genauen Angaben machen konnte, gelangte sie am Ende des Krieges zu der damals in Erfurt wohnenden Tochter Hedwig. Sie verbrachte ihre letzten Lebensjahre bei Heinz Hopf und seiner Frau in Zollikon, wo sie 1948 starb.

${ }^{7}$ Heinz Hopf hatte 1928 Anja (Annemarie) von Mickwitz (1891-1967) geheiratet. Sie war die Tochter eines evangelischen Pastors aus dem Baltikum.

${ }^{8}$ Berlin Südende, Langestraße 18.

${ }^{9}$ Hedwig war zuerst mit Richard Lachmann (1885-1916; im Kriegsdienst umgekommen) verheiratet. Die Tochter Elisabeth aus dieser Ehe studierte ab 1934 in Zürich und Basel Kunstgeschichte und Archäologie; sie promovierte 1942 in Basel. 1940 hatte sie Leopold Ettlinger geheiratet, von dem weiter unten die Rede sein wird, siehe Fußnote 12. In zweiter Ehe war Hedwig Hopf mit Fritz Brinckmann verheiratet (1929). Aus dieser Ehe entsprossen zwei Kinder, Ludwig und Marianne.
} 
dem 3. Januar in Berlin. Auf dem Rückweg wollten sie am 9. Januar in Karlsruhe bei Emmy Ettlinger ${ }^{10}$ Station machen.

Auf dem Bahnhof Karlsruhe wurden Heinz und Anja (Annemarie) Hopf, wie auch Frau Emmy Ettlinger und die sie begleitende Tochter Theresa Hemmerdinger, von der Gestapo verhaftet. Das Einlieferungsprotokoll ins Gefängnis II in Karlsruhe vermerkt als Haftgrund ,Schutzhaft"“.11

Leopold Ettlinger ${ }^{12}$ (siehe Abb. 2), damals Student der Landwirtschaft an der ETH in Zürich, dem die Rückkehr von Heinz und Anja Hopf auf den 9. Januar angekündigt worden war, war über das Ausbleiben der beiden höchst beunruhigt. Er wandte sich am 11. Januar brieflich an Schulratspräsident Rohn und beschrieb die Situation. ${ }^{13}$ Er hatte bereits in Berlin und Karlsruhe telefonisch nachgefragt und vernommen, dass sich seine Mutter, Emmy Ettlinger, und seine verheiratete Schwester, Theresa Hemmerdinger, am Montag, den 9. Januar zum Bahnhof in Karlsruhe begeben hätten, um das Ehepaar Hopf zu treffen, dass sie danach aber nicht mehr nach Hause zurückgekehrt seien.

\footnotetext{
${ }^{10}$ Emmy Ettlinger, Karlsruhe, Baden, Schlieffenstraße 10. Emmy Ettlinger ist die Mutter von Leopold Ettlinger (siehe Fußnote 12), der in Zürich Agronomie studierte und dort die Nichte von Heinz Hopf, Elisabeth Lachmann kennen gelernt hatte. Die beiden heirateten 1940.

${ }^{11}$ Siehe [9]. - Das Instrument der ,Schutzhaft“" wurde im Dritten Reich 1934 eingeführt, um - wie damals euphemistisch behauptet wurde - die Betroffenen vor dem Volkszorn zu schützen. In Tat und Wahrheit war es von Anfang an, aber besonders nach dem verschärfenden Schutzhafterlass vom 25. Januar 1938, ein Instrument, das der Gestapo und anderen Stellen die willkürliche Verfolgung von missliebigen Personen erlaubte. - Das Einlieferungsprotokoll vermerkt bei Frau Hemmerdinger die Vornamen Theresa Charl(otte) Sarah. Mit der Verordnung vom 17. August 1938 wurden Juden gezwungen, zusätzlich die Vornamen Israel bzw. Sara(h) zu führen. Die Verordnung trat am 1. Januar 1939 in Kraft.

12 Leopold Ettlinger (1914-2008) kam 1935 aus Deutschland in die Schweiz, um an der ETH Agronomie zu studieren. 1938 wurde ihm in einer Kollektivaktion die deutsche Staatsbürgerschaft aberkannt. Danach zählte er in der Schweiz bis nach Kriegsende als Flüchtling (Toleranzbewilligung). Er erhielt 1939 das Diplom als Ingenieur Agronom, und 1945 wurde er promoviert. Im Jahr 1951 erwarb er mit seiner Frau und seinen beiden Kindern das Schweizerische Bürgerrecht. Von 1958 bis 1982 war er dann ordentlicher Professor an der ETH, zuletzt für Mikrobiologie. - Diese Informationen stammen zum Teil aus einem Beitrag von Leopold Ettlinger in [11, S. 49-54]. Darin berichtet Leopold Ettlinger über seine Erfahrungen in den Arbeitslagern, in welche die damals in der Schweiz lebenden Internierten und Flüchtlinge für kürzere oder längere Zeit einberufen wurden, um bei Meliorationen und Straßenbauten mitzuhelfen. Die Schweizer hatten während des Krieges ausgedehnten Militärdienst zu leisten. Wie die anderen Autoren dieses Buches wendet sich Leopold Ettlinger scharf gegen die generellen Angriffe, die Ende der 90er Jahre vom Simon Wiesenthal Center und anderen Stellen im Zusammenhang mit diesen Lagern gegen die Schweiz erhoben worden sind. Zur Konstruiertheit jener Vorwürfe vergleiche man auch [12, S. 350]. Der Kontakt von Leopold Ettlinger mit dem Ehepaar Hopf - und mit seiner späteren Ehefrau Elisabeth Lachmann - begann mit einem „Höflichkeitsbesuch“: wegen gemeinsamer Bekannten der Familien Hopf und Ettlinger hatte die Mutter Ettlinger dem jungen Studenten aufgetragen, in Zürich bei der Familie Hopf vorzusprechen. Während des Studiums war Leopold Ettlinger dann eng mit Hans Samelson befreundet. Samelson kam 1936 aus Breslau nach Zürich, um hier Mathematik zu studieren und insbesondere bei Heinz Hopf zu doktorieren. 1941 gelang ihm die Flucht via Spanien nach den USA, wo er später an der Stanford University tätig war (siehe [14]).

${ }^{13}$ Es ist denkbar, dass Leopold Ettlinger den Brief persönlich überbrachte; dies würde erklären, dass Rohn noch am selben Tag aktiv werden konnte.
} 


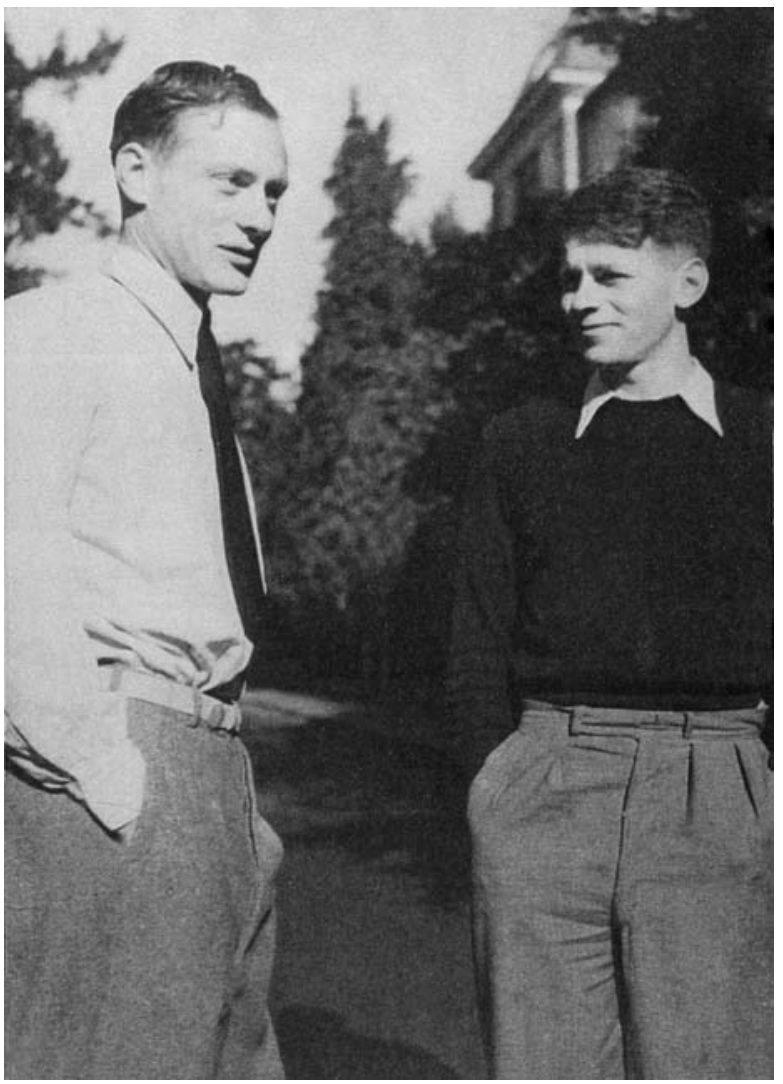

Abb. 2 Leopold Ettlinger (rechts) und Hans Samelson (links) während ihres Studiums in Zürich

Wohl fast gleichzeitig mit dem Brief von Leopold Ettlinger erreichte den Schulratspräsidenten eine an das Rektorat der ETH gerichtete handschriftliche Nachricht vom 10. Januar von Heinz Hopf aus Karlsruhe (siehe Abb. 3; [17], 221.2:102):

An das Rektorat der Eidgen. Technischen Hochschule.

Da ich zu meinem Bedauern meine Vorlesungen nach den Ferien nicht pünktlich beginnen konnte und auch jetzt voraussichtlich noch einige Zeit nicht werde nach Zürich kommen können, bitte ich für meine beiden Vorlesungen vorläufig eine Vertretung durch einen der Kollegen oder durch den Assistenten des Mathematischen Seminars einzurichten.

Hochachtungsvoll

Prof. Dr. H. Hopf

Die Handschrift zeigt die innere Erregung von Heinz Hopf deutlich. Die Tatsache, dass er überhaupt die Erlaubnis erhielt, aus der Schutzhaft einen Brief zu schreiben, muss wohl auf sein geschicktes Verhalten in dieser Situation zurückgeführt werden; offenbar verursachte sein Hinweis auf die wichtigen beruflichen Verpflichtungen in der Schweiz, die wahrzunehmen ihn die Verhaftung verhinderte, irgendwie Bedenken. 
238

U. Stammbach

16. I. $1939 \cdot 1$.

An das Refitorat der Eidgen. Techrsischen

Hochochnle.

Da ich in meinem Bedanern meine Vorlesungen nach den Ferien nicht punkwish begimmen konnte und anon jelte voranssichthichreimige teit micht werde nach zirrich hommen hiomen, bitte ich fir meine berden Vortesmque vorläntig eine Vertretung duroh einen der Herren Kolleger ader durch den Assiskenten des Mathematischen Perninars eimsurichten.

Hrchach tim govele

An den Norn Schutratsprüsidenten weitergeleitet.

Prof or. He Hort.

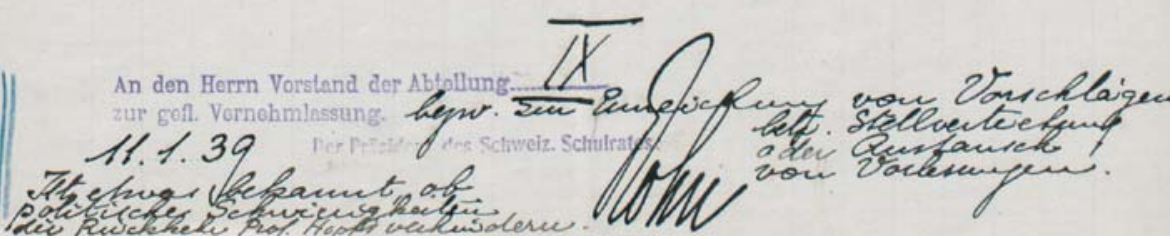

Abb.3 Brief von Heinz Hopf aus dem Gefängnis in Karlsruhe vom 10. Januar 1939 ([17], 1939-221_102)

Springer 


\section{Die Reaktionen in Zürich und Bern}

Schulratspräsident Rohn informierte noch am selben Tag die Abteilung für Mathematik und Physik ${ }^{14}$ und ließ anfragen: Ist etwas bekannt, ob politische Schwierigkeiten die Rückkehr von Prof. Hopf verhindern.

Rohn erkannte offenbar den Ernst der Situation. Gleichentags, also ebenfalls am 11. Januar, informierte er das Eidgenössische Politische Departement in Bern mit einem Brief an den vorstehenden Bundesrat ${ }^{15}$ über den Vorfall, und bat (siehe [15], 1939, 96/97):

Wir würden es für sehr angezeigt erachten, wenn Sie durch das Schweiz. Konsulat in Mannheim abklären lassen wollen, ob Herr Prof. Dr. Hopf in der Tat verhaftet worden sei und bis wann mit seiner Rückkehr nach der Schweiz gerechnet werden könne. Hierbei wäre allerdings dafür Sorge zu tragen, dass die Information des Studierenden Ettlinger den deutschen Behörden in keiner Weise bekannt wird, damit weder Herr Ettlinger selbst, nach dessen in Deutschland lebende Eltern hierdurch Unannehmlichkeiten ausgesetzt werden.

Das Politische Departement wurde sofort aktiv. Es teilt mit Schreiben vom 18. Januar dem Schulratspräsidenten mit, dass sowohl die Schweizerische Gesandtschaft in Berlin wie auch das Schweizerische Konsulat in Mannheim beauftragt worden seien, auf geeignetem Wege Erkundigungen über den gegenwärtigen Aufenthalt von Herrn und Frau Professor Dr. Hopf einzuholen und insbesondere abzuklären, ob die Genannten allenfalls in Deutschland verhaftet wurden. (Siehe [17], 221.2:227.)

Am 20. Januar erreicht ein weiterer Brief von Heinz Hopf Schulratspräsident Rohn, den dieser am 16. Januar ${ }^{16}$ in Karlsruhe geschrieben hatte. (Siehe[17], 221.2:253; Orthographie wie Original.)

\section{Sehr verehrter Herr Präsident!}

Ich muss Ihnen die peinliche Mitteilung machen, dass ich mich - ebenso wie meine Frau - in Untersuchungshaft befinde. Wie lange diese dauern und was weiter erfolgen wird, weiss ich noch nicht.

Ich bitte Sie natürlich sehr, diese Mitteilung äusserst diskret zu behandeln; jedoch wäre ich Ihnen dankbar, wenn Sie meine nächsten Kollegen wissen liessen, dass Sie Nachricht von mir erhalten haben.

\footnotetext{
${ }^{14}$ Die Abteilung für Mathematik und Physik trug damals an der ETH die Verantwortung für die Forschung und die Lehre in diesen beiden Fachgebieten. Ihr gehörten alle Dozenten in Mathematik und Physik an. - Die nachfolgende Notiz findet sich auf dem oben zitierten Brief, siehe Abb. 3.

${ }^{15}$ Das Politische Departement war für die Außenbeziehungen der Schweiz verantwortlich. Sein Vorsteher war damals Bundesrat Marcel Pilet-Golaz. - Eine Kopie des Schreibens ging außerdem an das Eidgenössische Departement des Innern (Vorsteher Philipp Etter).

${ }^{16}$ Es geht aus den Gefängnisprotokollen aus Karlsruhe hervor, dass Heinz Hopf und seine Frau am 16. Januar aus dem Polizeigefängnis in ein anderes Gefängnis, wohl in ein gerichtliches, verlegt wurden. Dies würde bedeuten, dass an diesem Datum die Angelegenheit aus den Händen der Polizei/Gestapo in die Hände der gerichtlichen Behörden gelangte. Für Hopf dürfte dies Grund zu einer gewissen, wenn auch geringen Beruhigung gewesen sein. Allerdings fehlen die gerichtlichen Gefängnisbücher in Karlsruhe aus jener Zeit, so dass die Annahme nicht zweifelsfrei erhärtet werden kann. Der Name Heinrich Hopf tritt in den Unterlagen erst wieder am 18. Januar in Erscheinung; unter diesem Datum wurde der Eintritt in das Gefängnis III vermerkt.
} 


\section{[...]}

Mit hochachtungsvollen Grüssen

verbleibe ich

Ihr stets sehr ergebener

H. Hopf

Schulratspräsident Rohn informierte umgehend Hopfs Kollegen in der Abteilung für Mathematik und Physik sowie das Politische Departement in Bern von der Nachricht aus Karlsruhe. - Am 28. Januar konnte das Politische Departement über seine Bemühungen berichten (siehe [17], 221.2:374; Orthographie wie Original):

[...] die Schweizerische Gesandtschaft in Berlin [konnte] von Herrn Dr. Brinckmann, Schwager des Genannten, erfahren [...], dass Herr und Frau Professor Hopf sich des Versuchs einer Uebertretung der deutschen Devisenvorschriften schuldig gemacht haben und deshalb in Karlsruhe verhaftet wurden.

Das Schweizerische Konsulat in Mannheim ist beauftragt worden, sich mit dem Verteidiger der Inhaftierten, Herrn Rechtsanwalt Dr. Eduard Dietz in Karlsruhe, in Verbindung zu setzen und über den Stand des Verfahrens zu erkundigen.

Die Bemühungen der Verwandten und Freunde von Herrn und Professor Hopf werden wohl dahin gehen müssen zu versuchen, gegen Stellung einer Kaution ihre Freilassung zu bewirken.

Genaueres ergibt sich aus dem Bericht der Schweizerischen Gesandtschaft in Berlin, der diesem Brief beigelegt wurde. Darin werden Angaben mitgeteilt, die aus einer von der Gesandtschaft organisierten Unterredung mit Dr. Brinckmann stammen. Frau Hopf habe in seiner Abwesenheit von Frau Brinckmann ein Brilliantenhalsband entgegengenommen und sich gefälligkeitshalber bereit erklärt, dasselbe nach der Schweiz mitzunehmen. [...] Professor Hopf sei eine typische Professorennatur und auch Frau Professor Hopf habe es zweifellos ferngelegen, eine Schiebung eines Wertgegenstandes nach der Schweiz vorzunehmen. Frau Hopf sei eine äusserst hilfsbereite und gefällige Frau und habe damit irgend jemanden einen Gefallen erweisen wollen, wobei sie wahrscheinlich nicht einmal gewusst hat, wem das Halsband gehört. Dr. Brinckmann hat erst durch die Kriminalpolizei, die auch bei ihm eine Hausdurchsuchung vorgenommen hat, erfahren, dass das Halsband einem Bankhaus Homburger gehörte und vermutlich für einen jüdischen Studenten in der Schweiz bestimmt war. ${ }^{17}$ Dr. Brinckmann glaubt, Professor Hopf habe vielleicht überhaupt nichts von der Sache gewusst, sich jedoch als Kavalier vor seine Frau gestellt, womit der Fall wesentlich verschlimmert worden sei, weil die deutschen Behörden Frauen gegenüber bei solchen Delikten weniger scharf vorgehen als gegenüber Männern. ${ }^{18}$

\footnotetext{
${ }^{17}$ Es handelt sich um das Bankhaus Veit L. Homburger in Karlsruhe. Das Bankhaus wurde Anfang 1939 ,arisiert“. Siehe dazu den Epilog 2 am Ende dieses Beitrages und die zugehörigen Fußnoten. - Es kann spekuliert werden, dass das Halsband für den Sohn von Paul Homburger, Friedrich Rudolf, bestimmt war, der zu jener Zeit an der ETH in Zürich Maschinenbau studierte.

${ }^{18}$ Der letzte Satz ist vielleicht eine diplomatische Umschreibung der Tatsache, dass die Stellung von Heinz Hopf als „Halbjude“ die Angelegenheit besonders gefährlich machte. In einem offiziellen Papier der Gesandtschaft wollte man wahrscheinlich nicht explizit festhalten, dass sich die an sich neutral formulierte neue deutsche Verordnung vor allem gegen Juden richtete.
} 
Es seien neben dem Brilliantenhalsband noch zwei weitere kleinere Schmuckstücke in die Untersuchung einbezogen. Ferner sei eruiert worden, dass im Herbst des vorhergehenden Jahres eine Perlenkette in die Schweiz mitgenommen worden sei. Das Verhalten verstoße gegen das Gesetz über die Devisenbewirtschaftung vom 12. Dezember 1938; dieses halte in $\S 54$ fest: „Nur mit Genehmigung dürfen Sachen unentgeltlich ins Ausland oder aus dem Inland in die badischen Zollausschlussgebiete übersandt oder gebracht werden." Da vorher keine derartigen Bestimmungen existiert hätten, bedeute wahrscheinlich die Mitnahme des Perlencolliers im September 1938 keinen Verstoß gegen die damals herrschenden Devisenvorschriften. ${ }^{19}$

\section{Unterwerfungsverfahren und Entlassung}

Heinz Hopf und seine Frau wurden am 28. Januar aus der Haft entlassen, und sie reisten am Sonntag, den 29. Januar nach Zürich. Schulratspräsident Rohn hatte unmittelbar danach, am 30. Januar, eine Unterredung mit Heinz Hopf, in der dieser über die Angelegenheit berichtete. Rohn teilte den Inhalt dieser Besprechung gleichentags brieflich dem Politischen Departement in Bern mit. Sein Brief kreuzte sich mit dem Brief vom 2. Februar aus Bern, in dem das Politische Departement seine inzwischen erhaltenen Informationen aus Mannheim an Schulratspräsident Rohn weitergab. In diesem Schreiben wird aus dem Bericht des Anwaltes Dr. Dietz vom 30. Januar an das Schweizerische Konsulat in Mannheim zitiert (siehe [17], 221.2:442; Orthographie und Hervorhebungen wie Original):

In der Angelegenheit Professor Dr. Heinrich Hopf aus Zürich setze ich in Verfolg meines Schreibens vom 26. ds. in Kenntnis, dass es inzwischen gelungen ist, bereits am Samstag, den 28. Januar nachmittags, bezw. abends die Freilassung der Eheleute Hopf gegen Zahlung einer Geldstrafe von je RM. 3000.-zusammen also RM. 6000.-. zahlbar in Schweizer Franken, zu erwirken, nachdem durch die Vermittlung der Deutschen Bank, des Schweizerischen Bankvereins und der Schweizerischen Kreditanstalt in Zürich der erforderliche Frankenbetrag von sfrs. 10.741 .97 (einschliesslich hiesiger Bankspesen mit RM. $20.95+18 .-=$ RM. 38.95) an die hiesige Zollkasse hatte überwiesen werden können.

[...]

Die Verurteilung im Unterwerfungsverfahren erfolgte auf Grund der $\S \S 54$ und 69 des neuen Devisengesetztes wegen Versuchs, einige Schmuckstücke aus Gefälligkeit für Bekannte ohne die seit 1.I.1939 erforderliche devisenamtliche Genehmigung über die Grenze zu nehmen.

$[\ldots]$

\footnotetext{
${ }^{19}$ Im Laufe der Jahre 1938 und 1939 erließ das Dritte Reich in einem beschleunigten Rythmus immer striktere Verordnungen, die gezielt gegen den jüdischen Teil der Bevölkerung gerichtet waren. Das hier erwähnte Gesetz über die Devisenbewirtschaftung sowie die Verordnung, welche die Juden zwang, die Namen Israel bzw. Sara anzunehmen (siehe Fußnote 11), sind nur zwei dieser zahlreichen Erlasse. Zusätzlich erschwerte man durch verschiedene Vorkehren sukzessive die Emigration. So durften Vermögenswerte nur mehr zu einem geringen Anteil ins Ausland mitgenommen werden und der Staat erhob eine immer höhere Reichsfluchtsteuer.
} 
Ich glaube, mit den Nächstbeteiligten selber, die erzielte Erledigung als eine verhältnismässig günstige bezeichnen zu dürfen.

Am 1. Februar nahm Heinz Hopf seine Vorlesungstätigkeit an der Eidgenössischen Technischen Hochschule wieder auf.

\section{Private Hilfe aus der Schweiz}

Soweit der Ablauf dieser Angelegenheit, wie er sich aus den offiziellen Papieren ergibt. Es ist bemerkenswert, wie rasch und intensiv sich die damalige Schulleitung der Sache annahm und wie effizient das Politische Departement in Bern, die Schweizerische Gesandtschaft in Berlin und das Schweizerische Konsulat in Mannheim arbeiteten. Wir wissen nicht, ob die Wahl des Anwaltes ebenfalls auf Betreiben der Schweizerischen Stellen erfolgte. Aber Dr. Eduard Dietz (1866-1940) war eine damals sehr prominente Persönlichkeit. Er war nach dem ersten Weltkrieg als Vertreter der SPD maßgeblich an der Ausarbeitung der neuen republikanischen badischen Verfassung beteiligt gewesen. Er trat dann aber 1920 aus der SPD aus, gab seine politischen Ämter auf und beschränkte sich in den folgenden Jahren auf seine Anwaltstätigkeit. Von 1922 bis 1933 war er Vorsitzender der badischen Anwaltskammer. Es scheint, dass er nach der Machtübernahme der Nazis bis zu seinem Tode im Jahre 1940 den politischen Einflüssen weitgehend widerstanden hat.

Die Erledigung der Angelegenheit der Verhaftung des Ehepaares Hopf in Karlsruhe durch das Unterwerfungsverfahren - wie es sich aus den offiziellen Papieren ergibt - erscheint uns heute als eine für die Beteiligten einfache und ,günstige“ Lösung. Die direkt Betroffenen waren über den Ausgang sicher ebenfalls erleichtert, aber es ergibt sich aus Schriftstücken, die erst vor wenigen Jahren aufgetaucht sind, dass der ,,verhältnismässig günstigen Erledigung“ einige grössere Schwierigkeiten entgegen standen. ${ }^{20}$

Es geht aus diesen Schriftstücken hervor, dass Heinz Hopf sich am 23. Januar aus dem Gefängnis in Karlsruhe brieflich an Ernst Völlm in Zollikon wandte. Ernst Völlm hatte an der ETH bei Polya in Mathematik promoviert und sich danach auch habilitiert (siehe Abb. 4). Er gab über längere Zeit an der ETH Vorlesungen über graphische und numerische Rechnungsverfahren; 1948 wurde er zum Titularprofessor ernannt. Zusammen mit seiner Ehefrau besass er ein größeres Grundstück mit Seeanstoß in Zollikon. Dort fanden in den dreißiger Jahren viele Einladungen statt, an denen die Mathematiker der ETH teilnahmen, u. a. George Pólya, Walter Saxer und Heinz Hopf. Besonders eng gestaltete sich das Verhältnis mit dem ebenfalls in Zollikon wohnenden Ehepaar Hopf. Der handschriftliche Brief von Heinz Hopf an Ernst Völlm hat folgenden Inhalt (siehe [19], Orthographie und Hervorhebungen wie Original $^{21}$ )

\footnotetext{
${ }^{20}$ Herr Dr. Klaus Völlm, Sohn von Ernst Völlm, hat diese Dokumente im Nachlass seines Vaters gefunden und sie in verdankenswerter Weise dem Archiv der Bibliothek der ETH übergeben.

${ }^{21}$ Hopf benützt zu jener Zeit in seinen Briefen an Stelle von ß stets ss; in der Schweiz hat sich diese Abweichung von den Vorschriften nach Duden ab den 1930er Jahren durchgesetzt. Heute verzichten in der
} 


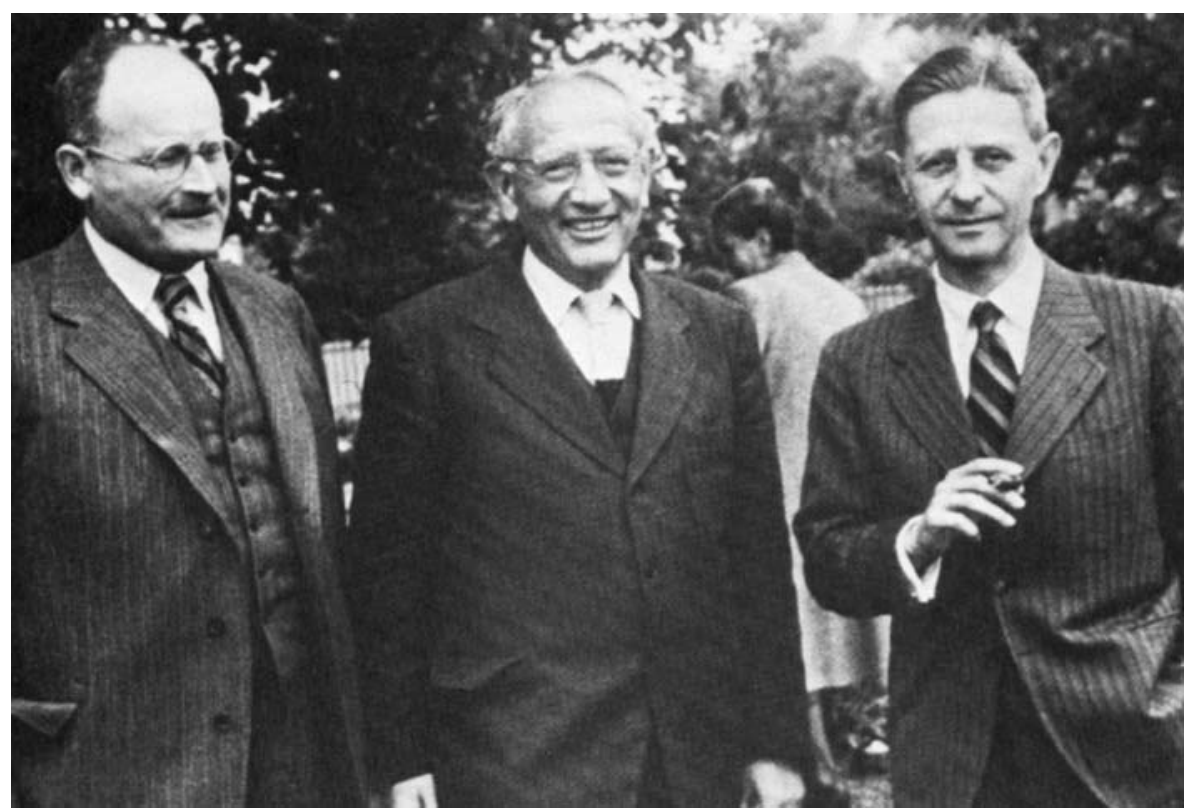

Abb. 4 Ernst Völlm, George Pólya, Heinz Hopf (von links nach rechts) im Garten des Anwesens von Ernst Völlm in Zollikon

\section{Lieber Herr Völlm!}

Ich bitte Sie erstens sehr herzlich, über diesen Brief mit niemanden - ausser natürlich Ihrer Frau - zu sprechen. Zweitens aber bitte ich Sie noch in einer anderen Richtung um ein ungewöhnlich grosses Vertrauen:

Meine Frau und ich sind am 9.I. im Zuge auf dem Bahnhof Karlsruhe von der Polizei festgenommen worden und seitdem in Haft. Gründe und Einzelheiten sind im Augenblick wohl nicht wesentlich. Wesentlich aber ist: einem Strafverfahren das langwierig und kostspielig wäre und das, was die Hauptsache ist, mit längeren Freiheitsstrafen zu enden droht, können wir dadurch, und nur dadurch, entgehen, dass wir uns einer hohen Geldbusse unterwerfen. Ich will, wenn irgend möglich die Unterwerfung wählen; denn abgesehen von der unmittelbaren Wirkung einer Freiheitsstrafe, besonders auch auf meine Frau, würde eine weitere mittelbare Wirkung wohl darin bestehen, dass die Fortsetzung meiner Tätigkeit in Zürich an der E.T.H. stark in Frage gestellt würde. Aber die Geldbusse, der ich mich also unterwerfen will, ist wiederum im Augenblicke dadurch unmöglich, als sie (da ich devisenrechtlich „Ausländer“ bin) in Schweizer Franken geleistet werden muss und mein Zürcher Guthaben die Höhe der zu leistenden Busse nicht erreicht. Die Summe, die mir fehlt, ist etwa 7000 Fr. Ich bin mir der Ungewöhnlichkeit des Unterfangens vollkommen bewusst, wenn ich Sie kurz und bündig bitte und frage: ,Können Sie mir 7000 Fr. leihen, und zwar sofort?“ - Da ich kein Haus besitze, kann ich Ihnen keine Hypothek anbieten; (an Sicherheiten materieller Art könnte ich Ihnen also nichts als etwa unsere Möbel und den Schmuck

Schweiz die Zeitungs- und Buchverlage wie zum Beispiel der NZZ-Verlag durchwegs auf den Gebrauch von $B$. 
meiner Frau vorschlagen.) Jedenfalls verpflichte ich mich, bei jeder meiner vierteljährlichen Gehaltszahlungen, beginnend Frühjahr 39, je $300 \mathrm{Fr}^{22}$ zurückzuzahlen; ausserdem bitte ich Sie, einen Zinsfuss festzusetzen; evtl. könnte ich auch gleich nach meiner Rückkehr nach Zürich einen wesentlichen Teil der Schuld zurückzahlen, indem ich noch anderweitig eine Anleihe unterbringe. - Jedenfalls bitte ich Sie sehr, 1) so schnell wie möglich, evtl. telegraphisch Bescheid an Rechtsanwalt Dr. E. Dietz, Karlsruhe, Kaiserstr. 199 zu geben, ob u. wann Sie mir die Summe leihen können; 2) falls Sie sie mir sofort leihen können und wollen, die Summe auf mein Konto bei der Schweizerischen Kreditanstalt, Paradeplatz, Zürich einzuzahlen. Die Sache ist insofern eilig, als, sobald die ganze Busse gezahlt ist (die ich von der Kreditanstalt hierher überweisen lassen würde), wir sofort in Freiheit gesetzt werden sollen und die ganze Sache erledigt sein soll, so dass wir heimkehren können. - Falls Sie selbst nicht in der Lage sind, mir das Geld zu leihen, so könnten Sie mir vielleicht, durch Herrn Dr. Dietz, einen Rat geben, was man tun könnte.

Gesundheitlich geht es mir und, wie ich hoffe, auch meiner Frau gut. ${ }^{23}$

Mit herzlichen Grüssen ,von Haus zu Haus“

Ihr Heinz Hopf

Der Ton des Briefes ist für Heinz Hopf ganz unüblich, es besteht deshalb kein Zweifel, dass Ernst Völlm den Ernst der Lage klar erkennen musste. Er entsprach denn auch der Bitte umgehend, so dass am 27. Januar von Dr. Dietz bereits die Bestätigung des Empfanges der 7000 Franken vorlag. Hopf konnte am 26. Januar an Völlm telegraphieren (siehe [19]):

\section{voellm seestrasse 41 zollikon $=$ danke $=$ hopf}

Die Summe von Fr. 10.000.-, die Hopf als Buße zu bezahlen hatte, war für ihn von beträchtlicher Höhe: sie entsprach ungefähr der Hälfte seines Jahresgehalt, das er damals als ordentlicher Professor an der ETH erhielt. Es geht aus weiteren Unterlagen hervor, dass Ernst Völlm die Summe von 4000 Franken als zinsloses Darlehen gewährte, während ihm Hopf gleich nach der Rückkehr 3000 Franken zurückzahlte.

Es wird aus dem Ablauf deutlich, dass diese Verhaftung den deutschen Stellen vor allem dazu diente, Schweizerische Devisen zu beschaffen. Insbesondere durfte Hopf nicht auf seine eigenen Vermögenswerte und die seines Vaters zurückgreifen, die noch in Deutschland lagen. Aus den Unterlagen geht auch hervor, dass man versuchte, von Frau Hopf Auskünfte über die Vermögenslage in der Schweiz zu erhalten. Durchaus möglich ist ferner auch, dass Hopf im Brief an Ernst Völlm mit Absicht als monatliche Zurückzahlung zuerst Fr. 500.- einsetzte und diese dann auf Fr. 300.- reduzierte. Damit versuchte er vielleicht gegenüber den mitlesenden deutschen Stellen zu signalisieren, dass der Betrag von Fr. 300.- für ihn das äußerst Mögliche war. ${ }^{24}$

\footnotetext{
${ }^{22}$ Der zuerst eingesetzte Betrag von 500 Fr. hat Heinz Hopf durchgestrichen, aber so, dass er lesbar blieb.

${ }^{23}$ Man hatte Heinz Hopf erlaubt, während der Haft mit seiner Frau drei kurze handschriftliche Notizen auszutauschen. Siehe [7], Hs 621:1636-1638.

${ }^{24}$ Es sind Fälle bekannt, wo von deutscher Seite auf ähnliche Art versucht wurde, an private Vermögenswerte heranzukommen, die sich im Ausland, insbesondere in der Schweiz befanden. Dabei wurden typischerweise Personen in ,Schutzhaft“ genommen und dann unter Druck gesetzt, ihre Vermögenswerte nach Deutschland zu transferieren. Siehe dazu [8, p. 104 ff.]
} 


\section{Bericht von Hopf an Alexandroff}

Es scheint, dass Hopf in der Folge von den Einzelheiten der gefährlichen Geschehnisse in Karlsruhe wenig erzählt hat. Sein Bekanntenkreis wusste nur, dass es einen Zwischenfall mit der Gestapo gegeben hatte, aber Genaueres war in seinem Umfeld offenbar nicht bekannt. Er hat wohl seinen Bekannten nur ähnlich viel verraten, wie er seinem engsten Freund Paul Alexandroff ${ }^{25}$ in Moskau in einem Brief vom 3. März 1940 kund tat. ${ }^{26}$ Um die Briefzensur - in der Zeit des Hitler-Stalin-Paktes musste auch im Briefverkehr mit Moskau mit Zensurmaßnahmen gerechnet werden - zu unterlaufen, versteckte er die wesentliche Nachricht zwischen mathematischen Ausführungen. Dabei verfremdete er zusätzlich die Geschichte noch in einer solchen Weise, dass sie heute ohne Wissen um die Hintergründe kaum mehr verständlich ist.

Uns geht es gut; (lediglich vor etwas mehr als einem Jahr hatte uns auf der Rückreise aus den Weihnachtsferien, die wir bei meinen Eltern verbracht hatten, eine böse Grippe überfallen, wodurch wir zu einem Aufenthalt in einem unangenehmen Sanatorium gezwungen wurden, der obendrein so kostspielig war, dass wir noch jetzt Schulden haben; aber hiervon abgesehen ist die Krankheit überstanden.)

Und nach weiteren mathematischen Ausführungen fügt er eine Seite später an:

Meine Eltern leben noch in Breslau, sehr einsam und unter Depressionen leidend; [...] wir waren längere Zeit nicht mehr bei ihnen; die Gefahr einer ähnlichen Erkrankung wie im vorigen Jahr ist auf einer so weiten Reise und, da wir uns seit vorigem Jahr etwas anfällig fühlen, nicht von der Hand zu weisen.

\section{Einbürgerung von Heinz und Anja Hopf in der Schweiz}

Obschon Heinz Hopf zusammen mit seiner Ehefrau seit 1931 in der Schweiz Wohnsitz hatte, hatte er bis 1943 kein Gesuch um Einbürgerung gestellt. ${ }^{27}$ Über die Gründe gibt ein Brief vom 14. April 1943 von Heinz Hopf an Schulratspräsident Rohn Aufschluss. Darin spricht er vom langjährigen Wunsch, Schweizerbürger zu werden; da er dauernd an der Eidgenössischen Technischen Hochschule wirken möchte, sei die bürgerrechtliche Verbundenheit mit der Schweiz auch der natürlichere Zustand. Der frühest mögliche Zeitpunkt für ein entsprechendes Gesuch wäre nach der damaligen Rechtslage nach einem zehnjährigen Aufenthalt in der Schweiz, also 1941, gewesen. Hopf sagt, er habe damals keinen derartigen Schritt unternommen,

und einen solchen auch weiterhin aufgeschoben, weil es mir schien, dass die unnormalen Zeiten einem solchen Gesuch nicht günstig seien, und weil ich daher glaubte, es sei klüger, normale Zeiten abzuwarten; besonders fürchtete ich den in solchem Zusammenhang häufig geäusserten Vorwurf des reinen Opportunismus. Jetzt

\footnotetext{
${ }^{25} \mathrm{Zu}$ Paul Alexandroff siehe [4, 5].

${ }^{26}$ Siehe [1], Hs 243:126.

${ }^{27}$ Die Unterlagen zur Einbürgerung von Heinz Hopf, aus denen in diesem Abschnitt zitiert wird, befinden sich unter [7], Hs 622:45-75.
} 
scheint es sich aber herauszustellen, dass dieses Zögern ein Fehler war. - Ich halte es nämlich neuerdings für wahrscheinlich, dass mir demnächst die deutsche Reichsangehörigkeit entzogen werde; diese Vermutung beruht auf einer Mitteilung, die ich vor drei Wochen von den juristischen Verwalter meines Vermögens in Deutschland erhielt und die besagt, dieses Vermögen sei - wie dem Herrn telephonisch und ohne Begründung von der Behörde bekanntgegeben wurde - beschlagnahmt worden; auf meinen sofort eingelegten Protest und mein Ersuchen um Begründung habe ich bisher keine Antwort erhalten; ich glaube aber, dass diese Massnahme nichts anderes bedeuten kann als meine Ausbürgerung aus Deutschland, die ihren endgültigen Ausdruck darin finden wird, dass meine deutschen Ausweispapiere bei nächster Gelegenheit nicht mehr erneuert werden. Die Erklärung wäre die, dass ich Halbjude bin; bekanntlich haben seit Ende 1941 alle im Ausland lebenden deutschen Juden die deutsche Reichsangehörigkeit verloren, und zwar vollzog sich dies, ohne weitere Mitteilung an die Betroffenen, in der Form, dass erstens ihr etwa noch in Deutschland befindliches Vermögen konfisziert wurde und zweitens ihre Ausweispapiere von den Konsulaten nicht verlängert wurden; Halbjuden hat man, soviel ich weiss, verschieden behandelt: die einen rechnete man zu den Juden, die anderen zu den Ariern; und die Beschlagnahme meines Vermögens kann ich nur dadurch erklären, dass man jetzt in Bezug auf mich die Entscheidung getroffen hat.

[...]

Dabei befinde ich mich in der peinlichen Lage, dass nun gerade etwas eintritt, was ich durch mein Zögern zu vermeiden hoffte: die Verteidigung gegen den Vorwurf des Opportunismus ist unter den gegebenen Umständen für mich schwieriger, als sie es früher gewesen wäre.

Hopf bat in diesem Brief am Schluss Schulratspräsident Rohn um Beratung und um Hilfe bei eventuell auftretenden Schwierigkeiten. Dieser setzte sich offenbar unmittelbar mit dem Chef der Eidgenössischen Fremdenpolizei, Heinrich Rothmund, in Verbindung, der mit Brief vom 30. April mitteilte, dass zwar die Antwort nur ganz unverbindlich und unbestimmt sein könne, dass aber rechtlich kein Hindernis auszumachen sei. Darauf reichte Heinz Hopf am 5. Mai für sich und seine Frau das Gesuch um Erteilung der Bewilligung zur Erwerbung eines Gemeinde- und Kantonsbürgerrechtes bei der Polizeiabteilung des eidgenössischen Justiz- und Polizeidepartementes ein. Er gab darin Schulratspräsident Rohn, seine Kollegen an der ETH, Walter Saxer und Michel Plancherel sowie Ernst Völlm als Referenz an. ${ }^{28}$

Die Befürchtung, die Hopf im Brief an Rohn geäußert hatte, dass ihm die deutsche Reichsangehörigkeit entzogen werden könnte, bewahrheitete sich bereits am 28. Mai 1943. An jenem Tag sprach Heinz Hopf beim Deutschen Generalkonsulat vor, um sich seinen Heimatschein erneuern zu lassen. Das Gespräch mit dem dortigen Beamten hielt Hopf wohl unmittelbar nach seinem Besuch schriftlich fest.

Ich gebe dem Beamten meinen Heimatschein, der am 17.VI.43 abläuft, mit der Bitte, ihn erneuern zu lassen. Der Beamte: ,Sind Sie Mitglied der deutschen Kolonie?“ Ich: „Nein.“ Er: ,,Warum nicht? Werden Sie nicht aufgenommen?“ Ich:

\footnotetext{
${ }^{28}$ Es ist nur natürlich, dass Heinz Hopf es jeweils sofort erfuhr, wenn die Fremdenpolizei diese Personen kontaktierte und befragte.
} 
„Ich bin halbarisch.“ Er: „Sind Sie Mischling ersten Grades?“ Ich: „,Mein Vater war Jude. “ Er: „Dann sind Sie Mischling ersten Gades. Und Ihre Frau? “ Ich: „Ist arisch.“ Er: „,Haben sie einen Pass?“ Ich: ,Ja.“ Er: „Ihr Heimatschein kann nicht erneuert werden. Ich habe Sie aufzufordern, in das deutsche Reichsgebiet überzusiedeln; im Weigerungsfalle haben Sie mit dem Verlust der deutschen Staatsangehörigkeit zu rechnen. ${ }^{29}$ Bitte äussern Sie sich dazu schriftlich. “ Ich: ,,Können Sie mir diese Aufforderung schriftlich zustellen?“ Er: „,Nein. “ Ich: „Dann bitte ich Sie, die Aufforderung noch einmal zu wiederholen, damit ich sie aufschreiben kann. " Er wiederholte wörtlich den oben unterstrichenen Satz - der dem Ton nach, in dem er gesagt wird, eine feststehende Formel zu sein scheint - und ich schreibe mit. Ich: ,Ist dies eine neue Bestimmung, die alle Mischlinge ersten Grades betrifft?“ Er: „Ja. “ Ich: „,Ich bin als Angestellter des Bundes durch einen Vertrag, der noch mehrere Jahre läuft, gebunden. “ Er (wörtlich): ,Es ist Ihre Sache, diese Schwierigkeiten zu meistern. " (Auch dies klang wie eine feststehende Formel.) Ich: ,Jedenfalls muss ich, bevor ich mich äussere, mit meinem Vertragspartner, also dem Bundesrat, in Verbindung treten. Daher wird bis zu meiner Äusserung vielleicht einige Zeit vergehen. “ Er: „,Bitte sehr.“ Ich: ,Kann ich meinen Heimatschein wieder mitnehmen?“ Er: „Nein, der bleibt hier."

Die „,rechtliche“ Basis für die Aufforderung an Heinz Hopf war die „Elfte Verordung zum Reichsbürgergesetz" vom 25. November 1941, welche sämtliche Juden und Jüdinnen im Ausland kollektiv die deutsche Staatsangehörigkeit absprach und gleichzeitig deren Vermögen beschlagnahmte. Unmittelbar nach seinem Besuch, bereits am 30. Mai, teilte Hopf dem Deutschen Generalkonsul brieflich mit, dass er entschlossen sei, im gegenwärtigen Augenblick nicht in das deutsche Reichsgebiet überzusiedeln. Er wiederhole nun auch formell den bereits mündlich ausgesprochenen Antrag, den Heimatschein zu erneuern.

Hopf informierte darauf Schulratspräsident Rohn von der dramatischen Entwicklung; rein rechtlich hätte der Verlust der deutschen Staatsangehörigkeit nämlich zur Konsequenz gehabt, dass Hopf das Niederlassungsrecht in der Schweiz hätte entzogen werden können. ${ }^{30}$ Rohn konnte versprechen, dass die Angelegenheit in der nächsten Schulratssitzung vom 25. Juni behandelt werde. Dort wurde beschlossen, das Einbürgerungsgesuch von Heinz Hopf vorbehaltslos zu unterstützen und sich gegebenenfalls auch direkt an den Vorsteher des Departementes des Innern, an Bundesrat Philipp Etter, zu wenden. ${ }^{31}$

Aus verschiedenen Gründen dauerte das Einbürgerungsverfahren recht lange. So gab es zum Beispiel Schwierigkeiten, die Geburtsurkunde von Frau Hopf beizubringen: Anja Hopf war 1891 in Pillistfer als Tochter eines evangelischen Pastors zur Welt gekommen. Damals war Pillistfer russisch, es wurde später estnisch, 1941 war

\footnotetext{
${ }^{29}$ Der hier hervorgehobene Satz ist im Original unterstrichen.

${ }^{30}$ Für eine Zusammenfassung der damals gültigen Gesetzesgrundlagen vergleiche man zum Beispiel [10, p. 26].

${ }^{31}$ Die deutschen Behörden verfolgten offenbar die Angelegenheit nicht intensiv weiter. Jedenfalls erhielt Heinz Hopf keine explizite Nachricht, dass er die deutsche Staatsangehörigkeit verloren hätte. Auf seine Nachfrage beim Deutschen Generalkonsulat in Zürich teilte man ihm am 24. September 1943 lediglich mit, dass sein Antrag den innerdeutschen Behörden unterbreitet worden sei, dass aber bis jetzt noch kein Bescheid eingegangen sei.
} 
es Kriegsgebiet geworden. Im Bericht des Pastors aus Pillistfer heisst es schließlich, dass die Kirchenbücher von den Sowjetrussen eingefordert worden seien und dass sie sich jetzt in Reval befänden. Mit Blick auf diese Schwierigkeiten waren die Schweizerischen Behörden bereit, das Gesuch auf der Grundlage des Passes von Anja Hopf weiter zu behandeln. Schließlich traf dann die Geburtsurkunde mit einem Brief vom 9. Juli der Direktion für Innere Verwaltung der Estnischen Verwaltung in Reval doch noch ein.

Auch ohne derartige Schwierigkeiten waren Bürgerrechtsverfahren komplex und dauerten lange, denn es waren die verschiedensten Behörden auf Bundes-, Kantonsund Gemeindeebene beteiligt, die alle ihre Zustimmung geben mussten. Immerhin wurde die in Hopfs Fall wohl wichtigste Hürde bereits am 15. Dezember 1943 genommen, als die Gemeindeversammlung in Zollikon in offener Abstimmung über das Gesuch positiv entschied. Schließlich wurde Heinz Hopf und seiner Frau mit Datum vom 26. Juni 1944 das Schweizerische Bürgerrecht verliehen; es machte ihn gleichzeitig zum Bürger der Gemeinde Zollikon und des Kantons Zürich.

\section{Epilog 1}

Der Zwischenfall in Karlsruhe lief für Heinz Hopf und seine Frau verhältnismäßig glimpflich ab. Es gibt viele Beispiele aus jener Zeit, die weit schlimmere Konsequenzen für die Betroffenen hatten. Für den günstigen Verlauf war zweifellos wichtig, dass Schulratspräsident Rohn die Nachricht des Studenten Ettlinger ernst nahm und sich sofort mit der Bitte um Hilfe an die Schweizerischen Politischen Behörden wandte. Ebenso wichtig war, dass die Behörden in Bern rasch und auf offenbar sehr effiziente Weise den Schweizerischen diplomatischen Apparat einschalteten; dies geschah, obschon Heinz Hopf und seine Frau damals noch nicht über das Schweizerische Bürgerrecht verfügten. Diese Handlungen verbunden mit den Bemühungen des Anwaltes Dr. Dietz in Karlsruhe haben in einem hohen Maß zu dem verhältnismäßig guten Ende beigetragen. Ganz besonders wichtig war schließlich die rasche und selbstlose Hilfe, die Heinz Hopf von Dr. Ernst Völlm in der Schweiz erfahren konnte, indem dieser Heinz Hopf von einem Tag auf den andern ein Darlehen in der Größenordnung von fast einem halben Jahresgehalt zur Verfügung stellte. ${ }^{32}$ Das Ehepaar Hopf selber, die Mathematiker an der ETH und die ETH als ganzes konnten dankbar auf den glimpflichen Ausgang dieses Zwischenfalles zurückblicken.

\footnotetext{
${ }^{32}$ Im Zuge der Auseinandersetzung um das Verhalten der Schweizer Banken während der Nazizeit wurden Zahlungen von Schweizer Banken nach Deutschland, die Konten von in Deutschland inhaftierten Personen betrafen, generell als illegitim angesehen, und die Nachkommen von Betroffenen wurden im Rahmen des Claims Resolution Tribunal entschädigt, und zwar auch dann, wenn die Umstände nur eine Vermutung einer solchen Zahlung zuließen. Man vergleiche dazu z. B. [2]. - Das im vorliegenden Beitrag beschriebene Beispiel zeigt, dass die Wahrheit manchmal komplizierter ist. - Zu weiteren Beispielen, aber auch zur Problematik derartiger Zahlungen vergleiche man [8, p. 48/49] sowie 104-120.
} 


\section{Epilog 2}

Im September 1954 erreichte Heinz Hopf ein Brief aus New York von Dr. Paul Homburger. ${ }^{33}$ Daraus sei der folgende Abschnitt zitiert:

[Ich] sehe es als eine moralische Verpflichtung an, zu meinem Teil fuer den materiellen Schaden aufzukommen, den Sie beim Versuch, uns zu helfen, erlitten haben. Das was Sie persönlich damals durchzumachen hatten, kann ja leider nicht aus der Welt geschafft werden, und es gibt keinen Ersatz hierfuer. Sie duerfen aber ueberzeugt sein, dass Ihre Hilfsbereitschaft in schwerster Zeit unter Einsetzung Ihrer eigenen Person von mir nie vergessen werden wird. ${ }^{34}$

Im nachfolgenden Brief vom 6. Oktober teilte Paul Homburger mit, dass er in allernächster Zeit den Betrag von Fr. 10.000.- zusätzlich zum Anwaltshonorar von rund Fr. 2500.- auf das Konto von Heinz Hopf überweisen werde.

Danksagung Der Autor dankt den folgenden Personen und Institutionen für Informationen zu den hier erwähnten Personen und Geschehnissen: Frau Dr. Elisabeth Ettlinger-Lachmann, Herrn Dr. Peter Ettlinger (Sohn von Leopold Ettlinger), Dr. med. Klaus Völlm (Sohn von Ernst Völlm), Dr. Reinhard Siegmund-Schultze.

ETH-Bibliothek, Zürich, Archive und Nachlässe (Michael Gasser, Marion Wullschleger); Landesarchiv Baden-Württemberg, Generallandesarchiv Karlsruhe (Dr. Martin Stingl), Stadtarchiv Karlsruhe (Jürgen Schuhladen-Krämer), Dokumentationsstelle Jüdische Zeitgeschichte im Archiv für Zeitgeschichte, ETH Zürich (lic. phil. Noëmi Sibold, Dr. Uriel Gast).

\section{Literatur}

1. Briefe von Heinz Hopf an Paul Alexandroff. ETH-Bibliothek, Archive und Nachlässe, Hs 243:95-147

2. In re Holocaust Victim Assets Litigation, Case No. CV96-4849. Certified Award in re Account of Veit L. Homburger, Claim Number: 224038/CH. Siehe http:/www.crt-ii.org/_awards/_apdfs/ Homburger_Veit.pdf, Letzter Zugriff: 28.5.2009

3. Eckmann, B. (Hrsg.): Heinz Hopf, Gesammelte Werke, Collected Works. Springer, Berlin Heidelberg New York (2001)

4. Frei, G., Stammbach, U.: Pawel Sergejewitsch Alexandroff, 1896-1982. DMV Mitteilungen 3, 17-22 (1996)

\footnotetext{
${ }^{33}$ Siehe [7], Hs 621:719-720. - Dr. Paul Homburger (1882-1968) war in den dreißiger Jahren Mitinhaber des Bankhauses Veit L. Homburger. Das Bankhaus wurde Anfang 1939 arisiert, nachdem die Inhaber anlässlich der Reichspogromnacht verhaftet und ins Konzentrationslager Dachau gebracht worden waren; Paul Homburger war dort bis zum 7. Dezember inhaftiert. Der hier beschriebene Vorfall fand also nur wenige Wochen nach seiner Entlassung aus Dachau statt. Im Zuge der Deportation der badischen Juden am 22. Oktober 1940 kam er dann ins Lager Gurs in Südfrankreich, damals Vichy-Frankreich. Von dort konnte er im März 1941 via Marseille in die USA gelangen. Es geht aus seinem Brief an Heinz Hopf hervor, dass Paul Homburger im Rahmen der Wiedergutmachungsverfahren der Bundesrepublik Deutschland Entschädigungen erhalten hatte. Er starb 1968 in New York.

${ }^{34}$ Die Umschreibung der Umlaute gemäß Original. - Aus der Einleitung dieses Briefes geht hervor, dass Paul Homburger kurz vorher mit Emmy Ettlinger Kontakt hatte und dass diese ihn darauf hingewiesen hatte, dass Heinz Hopf keine Aussicht hätte, im Wiedergutmachungsverfahren der Bundesrepublik Deutschland in dieser Sache eine Entschädigung zu erhalten. - Emmy Ettlinger (1882-1960) wurde am 22. Oktober 1940 ebenfalls nach Gurs deportiert. Sie konnte auf unbekannte Weise aus dem Lager fliehen und in Frankreich untertauchen. Nach Ende des Krieges kam sie in die Schweiz und lebte bis 1950 bei der Familie ihres Sohnes Leopold Ettlinger. Danach zog sie nach Palästina (Israel), wo auch ihre beiden Töchter, Therese Hemmerdinger und Hannah David, nach dem Krieg lebten.
} 
5. Frei, G., Stammbach, U.: Correspondence between Aleksandrov and Hopf 1926-1971. In: Topology and Applications, International Topological Conference dedicated to P. S. Alexandroff's 100th birthday, pp. xxiii-xxxviii. Phasis, Moscow (1996)

6. Frei, G., Stammbach, U.: Heinz Hopf (1894-1971). In: James, I. M. (Ed.) History of Topology, pp. 991-1008. Elsevier, Amsterdam (1999)

7. Hopf, H.: Nachlass, ETH-Bibliothek, Archive und Nachlässe, Hs 620-622

8. Jung, J. (Hrsg.): Zwischen Bundeshaus und Paradeplatz. Die Banken der Credit Suisse Group im Zweiten Weltkrieg. NZZ Verlag, Zürich (2001)

9. Landesarchiv Baden-Württemberg, Generallandesarchiv Karlsruhe. Polizeiliche Gefängnisbücher Gefängnis II, Gefangenenbuch 1939, (520 Zugang 1997-95/7, S. 12/23, Nr. 31 und 32), Gefängnis III, Gefangenenbuch 1938/39 (520 Zugang 1997-95/77, Nr. 132)

10. Ludwig, C.: Die Flüchtlingspolitik der Schweiz seit 1933 bis zur Gegenwart. Bericht an den Bundesrat zuhanden der eidgenössischen Räte (1957)

11. Newman, K.: Swiss Wartime Work Camps. NZZ Verlag, Zürich (1999)

12. Maissen, T.: Verweigerte Erinnerung. NZZ Verlag, Zürich (2005)

13. Pólya, G., Alexanderson, G. L. (Hrsg.): The Pólya Picture Album: Encounters of a Mathematician. Birkhäuser, Boston (1987)

14. Siegmund-Schultze, R.: Mathematiker auf der Flucht vor Hitler, Dokumente zur Geschichte der Mathematik, Band 10. Deutsche Mathematiker-Vereinigung, Vieweg (1998) [englische Ausgabe: Mathematicians Fleeing from Nazi Germany: Individual Fates and Global Impact. Princeton University Press (2009)]

15. Schulrat Missiven: ETH-Bibliothek, Archive und Nachlässe, SR1

16. Schulratsprotokolle: ETH-Bibliothek, Archive und Nachlässe, SR2

17. Schulratsakten: ETH-Bibliothek, Archive und Nachlässe, SR3

18. Stadtarchiv Karlsruhe: Projekt Gedenkbuch

19. Völlm, E.: Nachlass, ETH-Bibliothek, Zürich, Archive und Nachlässe, Nachträge zu Hs 1431.

20. Werner, J.: Hakenkreuz und Judenstern. Das Schicksal der Karlsruher Juden im Dritten Reich. Veröffentlichungen des Karlsruher Stadtarchivs, Bd. 9. Badenia Verlag, Karlsruhe (1988)

\section{Bildnachweis}

Abb. 1: Bild von Heinz Hopf, aus Selecta Heinz Hopf, Springer Verlag, 1964.

Abb. 2: Bild von Leopold Ettlinger und Hans Samelson, aus [14], Seite 113. Mit freundlicher Erlaubnis von Reinhard Siegmund-Schultze.

Abb. 3: Faksimile des Briefes von Heinz Hopf vom 10.1.1939. Mit freundlicher Erlaubnis der ETH-Bibliothek, Zürich, Archive und Nachlässe.

Abb. 4: Bild von Ernst Völlm, George Pólya, Heinz Hopf, aus [13], Seite 131. Mit freundlicher Erlaubnis von G. L. Alexanderson. 